\title{
COUNTABLE ENLARGEMENTS OF NORM TOPOLOGIES AND THE QUASIDISTINGUISHED PROPERTY
}

\author{
by F. X. CATALAN and I. TWEDDLE
}

(Received 3 January, 1992)

1. Introduction. Let $E$ be a Hausdorff locally convex space with continuous dual $E^{\prime}$ and let $M$ be a subspace of the algebraic dual $E^{*}$ such that $M \cap E^{\prime}=\{0\}$ and $\operatorname{dim} M=\aleph_{0}$. In the terminology of [4] the Mackey topology $\tau\left(E, E^{\prime}+M\right)$ is called a countable enlargement of $\tau\left(E, E^{\prime}\right)$. There has been some interest in the question of when barrelledness is preserved under countable enlargements (see [4], [5], [6], [8], [9]). In this note we are concerned with the preservation of the quasidistinguished property for normed spaces under countable enlargements; this was posed as on open question by $B$. Tsirulnikov in [7]. According to [7] a Hausdorff locally convex space $E$ is quasidistinguished if every bounded subset of its completion $\hat{E}$ is contained in the completion of a bounded subset of $E$ (equivalently, in the closure in $\hat{E}$ of a bounded subset of $E$ ). Any normed space is clearly quasidistinguished and remains so under a finite enlargement $\left(\operatorname{dim} M<\boldsymbol{\aleph}_{0}\right)$ since the enlarged topology is normable. (See the Main Theorem of [7] for a general result on the preservation of the quasidistinguished property under finite enlargements.) We shall write $Q D C E$ for a countable enlargement which preserves the quasidistinguished property.

For each infinite-dimensional normed space we can find a QDCE. A classical result of Grothendieck ([2, Corollaire 4 of Théorème 1]) shows that for a separable normed space every countable enlargement is a QDCE. However for a non-separable normed space of dimension $c$ (the cardinality of the real numbers), and more generally for a normed space with a $c$-dimensional non-separable quotient, there is always a countable enlargement which is not a QDCE. For its construction we make use of the space $\psi$ of [8], whose existence was established using the Continuum Hypothesis.

We are most grateful to the referee for pointing out an error in the first version of this note and for many valuable suggestions for improving the presentation of our results.

2. Preliminaries. We note first of all that, although our formulation of the problem is a little different from that in [7], the two versions are equivalent. The extended topology in [7] is defined as $\sup (\eta, \sigma(E, F))$, where $\eta$ is the given norm topology on $E$ and $F$ is a subspace of $E^{*}$ such that $E^{\prime}$ has codimension $\aleph_{0}$ in $F$. Since any metrizable space has its Mackey topology, we have $\eta=\tau\left(E, E^{\prime}\right)$. Also, $\sup (\eta, \sigma(E, F))$ has a countable base of neighbourhoods at the origin because of the codimensionality assumption and therefore it is the Mackey topology $\tau(E, F)$. Finally, if $M$ is any algebraic complement of $E^{\prime}$ in $F$, we have $\tau(E, F)=\tau\left(E, E^{\prime}+M\right)$.

Let $E$ be a normed space, let $\tau\left(E, E^{\prime}+M\right)$ be a countable enlargement of $\tau\left(E, E^{\prime}\right)$ and let $\left\{f_{n}: n \in \mathbb{N}\right\}$ be a basis in $M$. The Lemma of [7] allows us to identify the completion of $E\left(\tau\left(E, E^{\prime}+M\right)\right)$ with the product space $\hat{E}\left(\tau\left(\hat{E}, E^{\prime}\right)\right) \times \omega$, where $\hat{E}\left(\tau\left(\hat{E}, E^{\prime}\right)\right)$ denotes the completion of $E\left(\tau\left(E, E^{\prime}\right)\right)$ and $\omega$ is the topological product of countably many copies of the scalar field. This is achieved by identifying $E\left(\tau\left(E, E^{\prime}+M\right)\right)$ with the dense subspace $D=\left\{\left(x,\left(f_{n}(x)\right)\right): x \in E\right\}$ of $\hat{E} \times \omega$. We shall denote this identification by $\theta: E \rightarrow D$.

Glasgow Math. J. 35 (1993) 235-238. 
Now consider a bounded subset $A$ of $\hat{E} \times \omega$. If $\hat{B}$ denotes the closed unit ball of the Banach space $\hat{E}\left(\tau\left(\hat{E}, E^{\prime}\right)\right)$ we can find $k>0$ and a bounded subset $C$ of $\omega$ such that $A \subseteq k \hat{B} \times C$. Since $\{0\} \times C$ is a relatively compact subset of the metrizable space $\hat{E} \times \omega$, it is contained in the closed absolutely convex envelope of a sequence in $D$ converging to the origin (see [3, p. 134, Corollary]); in particular, $\{0\} \times C$ is contained in the completion of a bounded subset of $D$. It now follows that $D$ is quasidistinguished (under the topology induced by $\hat{E} \times \omega)$ if and only if $\hat{B} \times\{0\}$ is contained in the completion of a bounded subset of $D$. Since the quasidistinguished property is clearly preserved by a topological isomorphism, this condition is also necessary and sufficient for $\tau\left(E, E^{\prime}+M\right)$ to be a QDCE of $\tau\left(E, E^{\prime}\right)$.

The dual of $\omega$, which we denote by $\varphi$, is the direct sum of countably many copies of the scalar field. The $\sigma(\varphi, \omega)$-bounded sets are finite-dimensional.

3. The results. Throughout this section $E$ is a normed space and $\tau\left(E, E^{\prime}+M\right)$ is a countable enlargement of $\tau\left(E, E^{\prime}\right)$.

Proposition 1. If $E$ is separable, every countable enlargement of $\tau\left(E, E^{\prime}\right)$ is a $Q D C E$.

Proof. $E\left(\tau\left(E, E^{\prime}+M\right)\right)$ can be identified with a subspace of the separable metrizable space $E\left(\tau\left(E, E^{\prime}\right)\right) \times \omega$ and so it and each of its subsets are separable and metrizable. The result now follows from ([2, Corollaire 4 of Théorème 1]), where it is shown that every bounded separable subset of the completion of a metrizable space is contained in the closure of a bounded subset of the space.

We turn now to the existence of a countable enlargement which is not a QDCE. For this we make use of the space $\psi$ of [8], which is a dense barrelled subspace of $\omega$ with the property that each of its bounded sets spans a subspace with at most countable dimension.

Proposition 2. Assume the Continuum Hypothesis. If some (not necessarily Hausdorff) c-dimensional quotient of $E$ is non-separable, then there is a countable enlargement of $\tau\left(E, E^{\prime}\right)$ which is not a $Q D C E$.

Proof. Suppose that $E / G$ is non-separable and $c$-dimensional and let $H$ be any algebraic complement of $G$ in $E$. Since $\operatorname{dim} H=c$ we can find an algebraic isomorphism $t: H \rightarrow \psi$. Then $t^{\prime}: \varphi \rightarrow H^{*}$ and $t^{\prime-1}(X)$ is $\sigma(\varphi, \psi)$-bounded, where $X$ is the closed unit ball of $H^{\prime}$. It follows that $t^{\prime-1}\left(H^{\prime}\right)$ is a finite-dimensional subspace of $\varphi$. If $L$ is the subspace of $E^{*}$ obtained by extending each element of $t^{\prime}(\varphi)$ in such a way that it is identically zero on $G$, then $\operatorname{dim} L=\aleph_{0}$ and $L \cap E^{\prime}$ is finite-dimensional. Let $M$ be any subspace of $L$ which forms an algebraic complement of $E^{\prime}$ in $E^{\prime}+L$. We show that the countable enlargement $\tau\left(E, E^{\prime}+M\right)$ is not a QDCE.

Let $A$ be any non-empty bounded subset of $E\left(\tau\left(E, E^{\prime}+M\right)\right)$. Each element of $A$ can be uniquely expressed in the form $x_{1}+x_{2}$, where $x_{1} \in G, x_{2} \in H$. Let $A_{1}, A_{2}$ denote the sets of components in $G, H$ respectively of elements of $A$. Since each element of $L$ vanishes on $G$, it follows that such an element is bounded on $A_{2}$. Consequently $t\left(A_{2}\right)$ is $\sigma(\psi, \varphi)$-bounded and so $A_{2}$ must span an at most countable-dimensional subspace of $H$.

Using the identification described in Section 2, we have

$$
\theta(A) \subseteq \theta\left(A_{1}\right)+\theta\left(A_{2}\right) \subseteq G \times\{0\}+K
$$


for some countable-dimensional subspace $K$ of $H \times \omega$. Therefore

$$
\theta(A) \subseteq(G+N) \times \omega
$$

for some countable-dimensional subspace $N$ of $H$. Now, since $E / G$ is non-separable, it follows that $G+N$ is not dense in $E\left(\tau\left(E, E^{\prime}\right)\right)$. Consequently $\hat{B} \times\{0\}$ cannot be in the closure in $\hat{E} \times \omega$ of $\theta(A)$ for any bounded subset $A$ of $E\left(\tau\left(E, E^{\prime}+M\right)\right)$. This completes the proof.

Remark. Taking $G=0$ we see that Proposition 2 applies in particular to any non-separable $c$-dimensional normed space, e.g. $l_{\infty}$. Thus, assuming the Continuum Hypothesis, we have answered in the negative Tsirulnikov's question of whether every countable enlargement for any normed space is a QDCE.

We now give a sufficient condition for a countable enlargement to be a QDCE.

Proposition 3. If $E$ is the topological direct sum of $\mathrm{cl}\left(M^{\circ}\right)$ and a separable subspace, then $\tau\left(E, E^{\prime}+M\right)$ is a $Q D C E$ of $\tau\left(E, E^{\prime}\right)$.

Proof. Let $G=\operatorname{cl}\left(M^{\circ}\right)$ and let $E$ be the topological direct sum of $G$ and a separable subspace $H$. If $B_{1}, B_{2}$ are the closed unit balls of $G$ and $H$ respectively, we can assume without loss of generality that $B_{1}+B_{2}$ is the closed unit ball of $E$. The completion $\hat{E}$ of $E\left(\tau\left(E, E^{\prime}\right)\right)$ can be identified with the topological direct sum $\hat{G} \oplus \hat{H}$, where $\hat{G}$ and $\hat{H}$ are the completions of the normed spaces $G$ and $H$ respectively.

Following the procedure described in Section 2, we have

$$
\begin{aligned}
\hat{B} \times\{0\} & =\operatorname{cl}_{\hat{E}}\left(B_{1}+B_{2}\right) \times\{0\}=\left(\left(\operatorname{cl}_{\hat{G}} B_{1}\right) \oplus\left(\operatorname{cl}_{\hat{H}} B_{2}\right)\right) \times\{0\} \\
& =\left(\left(\operatorname{cl}_{\hat{G}}\left(B_{1} \cap M^{\circ}\right)\right) \oplus\left(\operatorname{cl}_{\hat{H}} B_{2}\right)\right) \times\{0\} .
\end{aligned}
$$

Since each element of $M$ vanishes on $B_{1} \cap M^{\circ}$, the set $\left(B_{1} \cap M^{\circ}\right) \times\{0\}$ is a bounded subset of the space $D$ of Section 2. Also, since $\tau\left(H, H^{\prime}+\left.M\right|_{H}\right)$ is a finite or countable enlargement of $\tau\left(H, H^{\prime}\right)$, it follows from the Introduction or Proposition 1 that the set $\left(\mathrm{cl}_{\hat{H}} B_{2}\right) \times\{0\}$ is contained in the closure of a bounded subset of $D$. Thus $\hat{B} \times\{0\}$ is contained in the closure of a bounded subset of $D$ as required.

Proposition 3 applies whenever $\mathrm{cl}\left(M^{\circ}\right)$ has finite codimension in $E$, the simplest case being that in which $M^{\circ}$ is dense in $E$. (This last condition has also been used in connection with the existence of barrelled countable enlargements ([1, Proposition 3.2 and Example 3.5], [4, Theorem 5], [6]).) As noted in the paragraph preceding Proposition 2 of [6], every infinite-dimensional normed space $E$ has a dense infinite-codimensional subspace $H$. If we choose for $M$ any $\aleph_{0}$-dimensional subspace of $E^{*}$ whose members vanish on $H$, we then have $M \cap E^{\prime}=\{0\}$ and $\operatorname{cl}\left(M^{\circ}\right) \supseteq \operatorname{cl}(H)=E$; consequently $\tau\left(E, E^{\prime}+M\right)$ is a QDCE. We have therefore established the following result.

\section{Proposition 4. Every infinite-dimensional normed space has a $Q D C E$.}

REMARK. In conclusion we note the following relevant facts concerning dimension. It is well known that a separable normed space has dimension at most $c$ and that an infinite-dimensional Banach space has dimension at least $c$ (without appeal to the Continuum Hypothesis). We are indebted to the referee for pointing out that any non-separable topological vector space must have an $\aleph_{1}$-dimensional non-separable subspace. Consequently a non-separable normed space of dimension greater than $c$ always has a non-separable $c$-dimensional subspace. 


\section{REFERENCES}

1. M. De Wilde and B. Tsirulnikov, Barrelledness and the supremum of two locally convex topologies, Math. Ann. 246 (1979-80), 241-248.

2. A. Grothendieck, Sur les espaces $(F)$ et $(D F)$, Summa Brasil. Math. 3 (1954), 57-123.

3. A. P. Robertson and W. J. Robertson, Topological vector spaces, second edition (Cambridge, 1973).

4. W. J. Robertson, I. Tweddle and F. E. Yeomans, On the stability of barrelled topologies, III, Bull. Austral. Math. Soc. 22 (1980), 99-112.

5. W. J. Robertson and F. E. Yeomans, On the stability of barrelled topologies, I, Bull. Austral. Math. Soc. 20 (1979), 385-395.

6. S. A. Saxon and W. J. Robertson, Dense barrelled subspaces of uncountable codimension, Proc. Amer. Math. Soc. 107 (1989), 1021-1029.

7. B. Tsirulnikov, On the locally convex noncomplete quasi-distinguished spaces, Bull. Soc. Roy. Sci. Liège 47 (1978), 147-152.

8. I. Tweddle, Barrelled spaces whose bounded sets have at most countable dimension, $J$. London Math. Soc. (2) 29 (1984), 276-282.

9. I. Tweddle and F. E. Yeomans, On the stability of barrelled topologies II, Glasgow Math. J. 21 (1980), 91-95.

Department of Mathematics

UNIVERSITY OF STRATHCLYDE

LIVINGSTONE TOWER

26 RichMOND STREET

GlasGow G1 1XH. 